Pacific Journal of Mathematic 


\title{
BASICALLY BOUNDED FUNCTORS AND FLAT SHEAVES
}

\author{
William C. Waterhouse
}

\begin{abstract}
A functor is here called basically bounded if, roughly speaking, it is determined by its values on objects of some bounded cardinality. For functors on $R$-algebras, it is shown that common constructions involving basically bounded functors can again be computed on algebras of bounded size, and hence are uniquely defined irrespective of any special set-theoretic assumptions. Even operations which seem to require arbitrarily large algebras-computing Čech cohomology and sheafifications in the flat topology, forming Ext groups and sheaves-turn out to be basically bounded. The proofs use homological algebra and a notion of approximation by small coverings.
\end{abstract}

In abstract algebraic geometry, e.g. [4], it is of ten convenient to embed the category of schemes in a certain category of functors known as the sheaves for the flat (fpqc) topology. This larger category has the advantage of containing cokernels. But unfortunately their construction involves sheafification, which requires taking a direct limit over all flat coverings. Such a limit has no obvious reason to exist and indeed may well not exist; as we shall see, functors in general simply do not have flat sheafifications. One can rescue the construction $a d$ hoc by restricting to a fixed "universe", but then the result will in general depend on the universe chosen. Yet all of this is unnatural; if we take an algebraic group acting on a variety over $\boldsymbol{Q}$, we never expect the quotient sheaf to have rational points whose existence depends on the size of the universe. It therefore ought to be possible to isolate the sheaves which have geometric meaning and deal with them in a way independent of arbitrary foundational assumptions. That is the purpose of this paper.

Much of the argumentation is category-theoretic. Indeed, the book of Gabriel and Ulmer [5], which came to my attention after this work was completed, overlaps part of the paper and provides a language for stating the results in extreme generality. In the interests of readability, however, I have chosen to present simply the detailed results for $R$-algebras and the flat topology. The basic special property of this topology is approximation by small coverings ( $(3)$, and with this in mind anyone who wants more general statements can easily abstract them. 
Throughout the paper $R$ will denote a fixed ring (commutative with unit), and $m$ will be an infinite cardinal not less than $\#(R)$. An $R$-algebra of cardinality at most $m$ will be called small. The category of $R$-algebras whose underlying sets are contained in some fixed set of cardinality $m$, say the von Neumann cardinal of that size, will be denoted $R$-Al $(m)$. Thus $R$-Al $(m)$ has in all $2^{m}$ objects and $2^{m}$ morphisms, and every isomorphism class of small $R$-algebras is represented in it. We use $j$ to denote the inclusion of $R-\mathrm{Al}(m)$ as a full subcategory of $R$-Al, the category of "all" $R$-algebras. It makes no difference how one makes sense of $R$-Al; it might be all $R$-algebras in a fixed universe, or simply $R$-Al $(n)$ for some $n$ bigger than $m$.

2. $m$-based functors. If $L$ is a (set-valued) functor on $R$-Al, then its restriction $j^{*} L$ is a functor on $R$-Al $(m)$. Left adjoint to $j^{*}$ is the Kan extension $j_{*}$, taking a functor $F$ on $R$-Al $(m)$ to a functor $j_{*} F$ on $R$-Al. Explicitly, $j_{*} F(A)$ is the direct limit of $F(S)$ over all morphisms $S \rightarrow A$ with $S$ in $R$-Al $(m)$. The injections $S \rightarrow A$ are obviously cofinal, and one can think of $j_{*} F(A)$ as the direct limit over all small subalgebras of $A$.

Definition. A functor on $R$-Al is called $m$-based if it is of the form $j_{*} F$. It is clear that this depends only on $m$, not on the particular set of cardinality $m$ underlying $R$-Al $(m)$.

TheOREM 2.1. Let $F$ denote a functor on $R$-Al $(m)$, and $L$ a functor on $R$-Al. Then

(a) $\operatorname{Hom}\left(F, j^{*} L\right)$ is naturally isomorphic to $\operatorname{Hom}\left(j_{*} F, L\right)$.

(b) The functor $j^{*} j_{*} F$ is naturally isomorphic to $F$.

(c) Every morphism of an m-based functor into $L$ factors uniquely through $j_{*} j^{*} L$.

(d) The m-based functors form a full subcategory of functors on $R$-Al; via $j^{*}$ and $j_{*}$ they are equivalent to the functors on $R$-Al $(m)$.

(e) $j_{*}$ preserves all direct limits.

(f) $j_{*}$ preserves monomorphisms and epimorphisms.

(g) $j_{*}$ preserves inverse limits over index categories having no more than $m$ morphisms.

Proof. Statements (a) through (e) are familiar general nonsense, (a) being the definition of left adjoint. Monomorphism and epimorphism of functors are tested at each value, so (f) means simply that the set-theoretic direct limits involved preserve injectivity and surjectivity. For surjectivity this is obvious. For injectivity 
it follows from the fact that the maps $S \rightarrow A$ form a good limit category, satisfying axioms $(L 1,2,3)$ of $[1, \mathrm{p} .7]$-a filtered category, in the language of $[6, \mathrm{p} .6]$.

To prove (g), finally, it is enough to show that $j_{*}$ preserves equalizers and preserves products having at most $m$ factors. Preservation of equalizers again follows trivially from the fact that maps $S \rightarrow A$ form a filtered category. Preservation of the products is a simple consequence of the additional fact that any $m$ maps $S_{i} \rightarrow A$ factor through a single map $S \rightarrow A$. (One obtains $S$ by choosing an algebra in $R$-Al $(m)$ isomorphic to the tensor product of the $S_{i}$.)

A functor to abelian groups we will call an abelian functor. Obviously abelian functors on $R$-Al $(m)$ have Kan extensions to abelian functors on $R$-Al, and one can repeat the preceding proof for them. Furthermore, since the index categories of maps $S \rightarrow A$ are filtered, the set-theoretic direct limits of abelian groups are the sets underlying the limit groups. Thus we have the following result.

THEOREM 2.2. (a) Let $F$ be an abelian functor on $R-\mathrm{Al}(m)$. The extension of $F$ to an abelian functor on $R$-Al has $j_{*} F$ as its underlying set functor; thus without ambiguity the extension can still be denoted $j_{*} F$.

(b) As a map from abelian functors on $R$-Al $(m)$ to abelian functors on $R-\mathrm{Al}$, the operation $j_{*}$ satisfies all the statements in (2.1).

Henceforth we will use Hom for abelian functor homomorphisms; arbitrary functor transformations, which will rarely occur, will be denoted Morph.

Definition. For $m \leqq n$ the $m$-based functors are a full subcategory of the $n$-based functors. The union of all these will be called the category of basically bounded functors on $R$-Al.

The rest of this paper is designed as evidence in favor of the following theses: (1) The basically bounded functors include most functors commonly occurring in algebraic geometry; and (2) Constructions involving basically bounded functors can be carried out on algebras of explicitly bounded size. For example, we have already verified (2) for direct and inverse limits over any specified index set. Some of the later proofs are not so easy, but the evidence still suggests that (1) and (2) will be true as a general rule. (As an explicit test, I have presented verifications for all constructions used in [7].) The basic claim, already stated in the 
introduction, is that ordinary abstract geometric constructions have no set-theoretic ambiguity: they are well defined and uniquely determined independent of any special foundational assumptions.

\section{Approximation by small coverings.}

Definition. Let $A$ be an $R$-algebra. A covering of $A$ for the flat topology is a finite family of morphisms $\left\{A \rightarrow B_{i}\right\}$ such that $A \rightarrow \Pi B_{i}$ is faithfully flat. (This is not saturated, i.e. in the terminology of $[6, p .14]$ it is a pretopology rather than a topology, but that makes no difference for our purposes.) A refinement of $\left\{A \rightarrow B_{i}\right\}$ is another covering $\left\{A \rightarrow C_{k}\right\}$ together with, for each $i$, an $A$-algebra map of $B_{i}$ into some $C_{k(i)}$.

Definition. Let $\left\{S \rightarrow T_{k}\right\}$ be a covering lying in $R$-Al $(m)$; for any map $S \rightarrow A$ the family $\left\{A \rightarrow A \otimes_{S} T_{k}\right\}$ is a covering of $A$. When $\left\{A \rightarrow B_{i}\right\}$ is a refinement of this covering, we call $\left\{S \rightarrow T_{k}\right\}$ a small covering approximation to $\left\{A \rightarrow B_{i}\right\}$. In other words, a small covering approximation is a small covering $\left\{S \rightarrow T_{k}\right\}$ together with maps $T_{k} \rightarrow B_{i(k)}$ lying over a single map $S \rightarrow A$.

Our results on small covering approximations rest on the following fact.

LEMma 3.1. Let $A \rightarrow B$ be faithfully flat, $S^{\prime}$ any small subalgebra of $A$, and $T^{\prime}$ any small subalgebra of $B$. Then there are small subalgebras $S \supseteqq S^{\prime}$ and $T \supseteqq T^{\prime}$ with $S \rightarrow T$ faithfully flat.

Proof. We view $A \rightarrow B$ as an inclusion. Recall [2, Chap. 1, $\S 3$, Prop. 13] that $A \rightarrow B$ is faithfully flat iff the following statement is true:

"Let $\Sigma_{k} y_{k} c_{k i}=d_{i}$ be any system of linear equations with the $c_{k i}$ and $d_{i}$ in $A$. Then every solution of the equations in $B$ has the form $y_{k}=x_{k}+\Sigma_{r} b_{r} z_{r k}$ where $\left(x_{k}\right)$ is a solution in $A$, the $b_{r}$ are in $B$, and for each $r$ the $\left(z_{r k}\right)$ are in $A$ satisfying the associated homogeneous equation $\Sigma_{k} z_{r k} c_{k i}=0 . "$

Now given $S^{\prime}$ and $T^{\prime}$ we first expand $T^{\prime}$ to make $T^{\prime} \supseteqq S^{\prime}$. Consider all the linear equations with $c_{k i}$ and $d_{i}$ in $S^{\prime}$; each solution in $T^{\prime}$ is a solution in $B$, and we write it in the form $x_{k}+\Sigma b_{r} z_{r k}$. Adjoin to $T^{\prime}$ all the $x_{k}, b_{r}$, and $z_{r k}$ needed for this, and let $T_{1}$ be the resulting algebra; then $T_{1}$ is again a small subalgebra. Set $S_{1}=T_{1} \cap A$. Apply the same process to $S_{1}$ and $T_{1}$, obtaining $S_{2}$ and $T_{2}$, and continue to iterate; let $T=\bigcup T_{n}$ and $S=\bigcup S_{n}=T \cap A$. Then $S \rightarrow T$ is faithfully flat. Indeed, consider any system of equations with entries in $S$ and any solution of it in $T$. The entries 
and solution then lie in some $S_{n}$ and $T_{n}$, and the elements needed to put the solution in the desired form are by construction available in $S_{n+1}$ and $T_{n+1}$.

Obviously this is a Skolem-Löwenheim argument.

Note that the lemma extends immediately to the case where we have a covering $\left\{A \rightarrow B_{i}\right\}$ with small subalgebras $S^{\prime} \subseteq A$ and $T_{i}^{\prime} \subseteq B_{i}$. For inside $B=\Pi B_{i}$ we find $T \supseteqq \Pi T_{i}^{\prime}$; and $T$, like $\Pi T_{i}^{\prime}$, will contain the idempotents of the product decomposition and thus will itself be a product $\Pi T_{i}$.

THEOREM 3.2. The small covering approximations to a fixed covering $\left\{A \rightarrow B_{i}\right\}$ form a filtered category in which every $m$ objects have an upper bound. Cofinal among them are those for which the index set is the same as for $\left\{A \rightarrow B_{i}\right\}$ and all the maps $S \rightarrow A$ and $T_{i} \rightarrow B_{i}$ are injective.

Proof. A morphism from $\left\{S^{\prime} \rightarrow T_{k}^{\prime}\right\}$ to $\left\{S \rightarrow T_{j}\right\}$ is of course given by consistent maps $S^{\prime} \rightarrow S$ and $T_{k}^{\prime} \rightarrow T_{j(k)}$ compatible with the maps to $A$ and $B_{i}$. For any small covering approximation $\left\{S^{\prime} \rightarrow T_{k}^{\prime}\right\}$ we can take the images of $S^{\prime}$ and $T_{k}^{\prime}$, expand them to a covering by the lemma, and choose an isomorphic covering in $R$-Al $(m)$. Hence the injective approximations with the same index set are cofinal. Obviously also any $m$ of them have an upper bound, since the union of their images can be expanded to a covering.

THEOREM 3.3. Let $\left\{A \rightarrow B_{i}\right\}$ be a covering. For each small approximation $\left\{S \rightarrow T_{i}\right\}$ with the same index set, and each finite family of indices $i_{1}, \cdots, i_{n}$, consider the induced map

$$
T_{i_{1}} \bigotimes_{S} \cdots \bigotimes_{S} T_{i_{n}} \longrightarrow B_{i_{1}} \bigotimes_{A} \cdots \bigotimes_{A} B_{i_{n}} .
$$

Then these maps are cofinal among maps of small algebras to $\bigotimes_{i=1}^{n} B_{i_{\lambda}}$, and thus for any m-based functor one can compute $j_{*} F\left(\otimes B_{i_{\lambda}}\right)$ as a direct limit over small covering approximations.

Proof. Observe first that by (3.1) the images of small covering approximations exhaust $A$ and $B_{i}$, and therefore the map from the direct limit of the $\otimes_{S} T_{i_{\lambda}}$ to $\otimes_{A} B_{i_{\lambda}}$ is surjective. An element of $\otimes T_{i_{\lambda}}$ which goes to zero in $\otimes B_{i_{\lambda}}$ does so because of some relation which involves only finitely many elements and so is captured in some small covering approximation; hence the limit is isomorphic to $\otimes B_{i_{\lambda}}$. 
Consider then any $\varphi: U \rightarrow \otimes B_{i_{\lambda}}$ with $U$ in $R$-Al $(m)$. Write $U$ as a quotient $L / I$ where $L$ is a polynomial algebra in $m$ indeterminates. Since $\varphi(U)$ is a small subalgebra, we can find an $\left\{S^{\prime} \rightarrow T_{i}^{\prime}\right\}$ such that the image of $\otimes T_{i_{\lambda}}^{\prime}$ includes $\varphi(U)$; and then the map $L \rightarrow U \rightarrow$ $\otimes B_{i_{\lambda}}$ factors through $\otimes T_{i_{\lambda}}^{\prime}$. Each element of $I$ goes to zero in some larger approximation; choosing $\left\{S \rightarrow T_{i}\right\}$ beyond all $m$ of these we get $\varphi$ factoring through $\otimes T_{i_{\lambda}}$.

4. Čech cohomology. If $\left\{A \rightarrow B_{i}\right\}$ is a covering and $L$ an abelian functor, recall [1, pp. 18-22] that the Cech cohomology groups $\check{H}^{n}\left(\left\{A \rightarrow B_{i}\right\}, L\right)$ are the homology groups of the sequence

$$
L(A) \longrightarrow \Pi L\left(B_{i}\right) \Longrightarrow \Pi L\left(B_{i_{1}} \otimes_{A} B_{i_{2}}\right) \Longrightarrow \cdots
$$

If $\left\{A \rightarrow C_{k}\right\}$ is another covering refining $\left\{A \rightarrow B_{i}\right\}$, then there are natural homomorphisms

$$
\check{H}^{n}\left(\left\{A \longrightarrow B_{i}\right\}, L\right) \longrightarrow \check{H}^{n}\left(\left\{A \longrightarrow C_{k}\right\}, L\right) .
$$

These are independent of the particular maps chosen to express the refinement. The direct limit over all coverings (if it exists) is denoted $\check{H}^{n}(A, L)$.

THEOREM 4.1. Let $F$ be an abelian functor on $R-\mathrm{Al}(m)$, and $\left\{A \rightarrow B_{i}\right\}$ a covering in $R$-Al. Let $\left\{S \rightarrow T_{k}\right\}$ run over the small covering approximations to $\left\{A \rightarrow B_{i}\right\}$. Then $\dot{H}^{n}\left(\left\{A \rightarrow B_{i}\right\}, j_{*} F\right)$ is the direct limit of the $\check{H}^{n}\left(\left\{S \rightarrow T_{k}\right\}, F\right)$.

Proof. Since the limit is taken over a filtered category, it is exact [1, p. 11]. Hence the result follows from (3.3).

Definition [1, p. 39]. An abelian functor $F$ is flasque if the cohomology groups $\check{H}^{n}\left(\left\{A \rightarrow B_{i}\right\}, F\right)$ vanish for all $n \geqq 1$ and all coverings.

CoRollary 4.2. If $F$ is a flasque abelian functor on $R$-Al (m), then $j_{*} F$ is also flasque.

THEOREM 4.3. Let $F$ be an abelian functor on $R-A l(m)$, and let $S$ be in $R$-Al $(m)$. Then $\check{H}^{n}\left(S, j_{*} F\right)$ exists and is naturally isomorphic to $\check{H}^{n}(S, F)$.

Proof. The first group is defined as the direct limit, over all $\left\{S \rightarrow B_{i}\right\}$, of $\check{H}^{n}\left(\left\{S \rightarrow B_{i}\right\}, j_{*} F\right)$. By (4.1), each of these is in turn 
the limit of $\check{H}^{n}\left(\left\{S \rightarrow T_{k}\right\}, F\right)$ over the small coverings approximating $\left\{S \rightarrow B_{i}\right\}$. Combining the two limits and recalling that refinement homomorphisms do not depend on the algebra maps giving the refinement, we see that we have simply the limit over all $\left\{S \rightarrow T_{k}\right\}$, that is, $\check{H}^{n}(S, F)$.

This elucidates a question arising in [3, pp. 67-70]. Working with Čech cohomology of $F(A)=A^{*}$ (Amitsur cohomology) and related functors, Chase and Rosenberg in effect replaced a limit over all coverings by a limit over small coverings. In their cases they could show that the groups so obtained turned out to be sufficiently large. Theorem 4.3 now shows that in fact they automatically had the full cohomology groups.

THEOREM 4.4. Let $F$ be an abelian functor on $R$-Al $(m)$. Then $\check{H}^{n}\left(-, j_{*} F\right)$ exists and is $m$-based, being in fact $j_{*}\left[\check{H}^{n}(-, F)\right]$.

Proof. Note first that for any $S \rightarrow A$, every small covering $\left\{S \rightarrow T_{k}\right\}$ approximates at least one covering of $A$, namely $\left\{A \rightarrow A \otimes_{S} T_{k}\right\}$. Now $\check{H}^{n}\left(A, j_{*} F\right)$ is by definition a direct limit over all $\left\{A \rightarrow B_{i}\right\}$, and by (4.1) each of these is in turn a limit over small approximations. Reversing the order of the limits we have the limit over all $S \rightarrow A$ of a limit over all small coverings $\left\{S \rightarrow T_{k}\right\}$, and that is $\left[j_{*} \check{H}^{n}(-, F)\right](A)$.

If $F$ is a functor whose values are nonabelian groups, the cohomology sequence does not exist but $\check{H}^{1}$ can be defined as a set. If $F$ is actually a sheaf of groups (to anticipate for a moment), then one can define other sheaves called principal homogeneous sheaves for $F$ over an algebra $A$; the definition does not matter here (cf. [4, Exp. IV, §5]), merely the fact that such objects are classified by $\check{H}^{1}(A, F)$. Restating the conclusions of (4.3) and (4.4) in this language gives us the following result.

THEOREM 4.5. Let $L$ be an m-based sheaf of groups.

(a) If $S$ is a small algebra, then every principal homogeneous sheaf for $L$ over $S$ is also m-based.

(b) Let $A$ be any algebra. Then each principal homogeneous sheaf for $L$ over $A$ comes from a principal homogeneous sheaf for $L$ over some small subalgebra of $A$.

Finally, if $F$ is an arbitrary functor, the equalizer sets $\check{H}^{0}\left(\left\{A \rightarrow B_{i}\right\}, F\right)$ and their limit $\check{H}^{\circ}(A, F)$ can still be defined, and 
the arguments still apply to them:

THEOREM 4.6. Let $F$ be any functor on $R$-Al $(m)$. Then the statements in Theorems 4.1, 4.3, and 4.4 are valid for $\check{H}^{0}$.

5. Sheaves and sheafification. Recall $[1, p .2]$ that a functor $L$ is a sheaf if the natural map $L(A) \rightarrow \check{H}^{0}\left(\left\{A \rightarrow B_{i}\right\}, L\right)$ is an isomorphism for every covering $\left\{A \rightarrow B_{i}\right\}$. Then Theorem 4.6 immediately yields the following basic result.

THEOREM 5.1. Let $F$ be a sheaf on $R$-Al $(m)$. Then $j_{*} F$ is a sheaf on $R$-Al.

Approximation by small coverings is of course the idea lying behind this theorem. Recall that if $L$ is any functor, a morphism $L \rightarrow M$ is the sheafification of $L$ if $M$ is a sheaf universal for morphisms of $L$ into sheaves. Combining (5.1) and $(2.1 a, c)$, we get:

CoRollary 5.2. Let $F$ be a functor on $R$-Al $(m)$ with sheafification $F \rightarrow G$. Then $j_{*} F \rightarrow j_{*} G$ is the sheafification of $j_{*} F$.

CoRollary 5.3. An m-based functor has a sheafification, and the sheafification is again m-based.

Proof. There is a standard construction, namely two applications of $F \mapsto \check{H}^{0}(-, F)$, which produces sheafifications [1, pp. 24-30]. On $R$-Al $(m)$ this process obviously can be performed without settheoretic difficulties, and we then use (5.2).

THEOREM 5.4. As a map from sheaves on $R$-Al $(m)$ to sheaves on $R$-Al, the operation $j_{*}$ satisfies all the statements of (2.1). The same is true of $j_{*}$ as a map on abelian sheaves.

ExAmple. To show that (5.3) has some point to it, we now exhibit a functor on $R$-Al which does not have a sheafification. For $P$ a prime ideal of an $R$-algebra $A$, let $\kappa(P)$ denote the fraction field of $A / P$. Let $F(A)$ be the collection of all locally constant functions $f$ which map the space $\operatorname{Spec}(A)$ into some von Neumann cardinal and satisfy $f(P)<\# \kappa(P)$ for all $P$. If $\varphi: A \rightarrow B$ is a homomorphism and $Q$ is a prime of $B$, then $\varphi^{-1}(Q)$ is a prime of $A$ satisfying $\# \kappa\left(\varphi^{-1} Q\right) \leqq \# \kappa(Q)$. Hence $\varphi$ does induce a map of $F(A)$ into $F(B)$, and $F$ is a functor. 
THEOREM 5.5. The functor $F$ just defined has no sheafification.

Proof. Let $n$ be any cardinal, and define $L_{n}(A)$ to be the locally constant maps from $\operatorname{Spec}(A)$ to $n$. This is a sheaf - in fact, it is given by the scheme which is a disjoint union of $n$ copies of Spec $(R)$. We map cardinals to $n$ by sending the elements less than $n$ to themselves and all others to zero; this gives a natural transformation $F \rightarrow L_{n}$. If $F$ has a sheafification $G$, the map $F \rightarrow L_{n}$ must factor through $G$. But for any given $A$ there is an $n$ for which $F(A) \rightarrow L_{n}(A)$ is injective; hence $F(A) \rightarrow G(A)$ must always be injective.

It follows from this that if $A \rightarrow B$ is faithfully flat, then the equalizer of $F(B) \rightrightarrows F\left(B \otimes_{A} B\right)$ must inject into $G(A)$. But suppose we let $A$ be a field and $B$ an extension field. The two set maps of $\operatorname{Spec}\left(B \otimes_{A} B\right)$ to $\operatorname{Spec} B$ are the same, since $\operatorname{Spec}(B)$ is a point; hence all of $F(B)$ must inject into $G(A)$. But $F(B)$ has the same cardinality as $B$, which can be arbitrarily large.

REMARKS. (a) The functor $F$ is obviously not basically bounded.

(b) The last half of the argument shows also that $\check{H}^{0}(A, F)$ does not exist.

(c) One can force the sheafification $G$ to exist by restricting to a fixed "universe", but then $G(A)$ will increase as the universe expands.

\section{Schemes.}

THEOREM 6.1. Let $L$ be the sheaf represented by an algebra $B$, i.e. $L(A)=\operatorname{Hom}_{R}(B, A)$. Then $L$ is $m$-based if and only if $\#(B) \leqq m$.

This is useful but trivial. Slightly more interesting is the following generalization, where the number of affines is irrelevant.

Theorem 6.2. Let $X$ be a scheme over Spec $(R)$ and $F$ the sheaf given by $X$, i.e. $F(A)=\operatorname{Hom}_{\operatorname{spec}(R)}(\operatorname{Spec}(A), X)$. If $X$ can be covered by open affines Spec $\left(B_{\alpha}\right)$ where each $\#\left(B_{\alpha}\right) \leqq m$, then $F$ is $m$-based.

Proof. Localizations of the $B_{\alpha}$ again have cardinality $\leqq m$; adding them to the collection, we may assume the Spec $\left(B_{\alpha}\right)$ are a basis of $X$. The result then follows from (5.4), (6.1), and the following lemma:

Lemma 6.3. Let $X$ be a scheme over Spec $(R)$, and $U_{\alpha}$ a basis 
of open subschemes. Then the sheaf given by $X$ is the sheaf direct limit of those given by the $U_{\alpha}$.

Proof. Let $Y$ be the functor direct limit of the sheaves given by the $U_{\alpha}$. If two elements $\operatorname{Spec}(A) \rightarrow U_{\alpha}$ and $\operatorname{Spec}(A) \rightarrow U_{\beta}$ give the same map to $X$, we can cover Spec $(A)$ by finitely many open affines Spec $\left(A_{\lambda}\right)$ each mapped to some $U_{\lambda} \subseteq U_{\alpha} \cap U_{\beta}$; the two elements of course give the same map on Spec $\left(A_{\lambda}\right)$. Since $\left\{A \rightarrow A_{\lambda}\right\}$ is a covering, the two elements are identified in the sheafification of $Y$, which thus injects into the sheaf given by $X$. That injection is surjective because, given any map $\operatorname{Spec}(A) \rightarrow X$, we can cover Spec $(A)$ by finitely many open affines each mapping into some $U_{\alpha}$.

Obviously the lemma is valid in the Zariski topology, not just the flat topology. This is perhaps the place to point out that our (flat) sheaves are indeed sheaves for the Zariski topology and hence extend in a unique reasonable way to (contravariant) functors on schemes over Spec $(R)$. This process defines an equivalence between our sheaves and the (fpqc) sheaves on schemes over Spec $(R)$, cf. [4, Exp. IV, §6]. The restriction to functors on $R$-algebras has been adopted purely as a matter of convenience.

\section{Derived functors and Ext groups.}

THEOREM 7.1. Let $\Sigma$ be a left-exact functor on abelian sheaves over $R-\mathrm{Al}(m)$, and let $R^{i}$ denote derived functors. Then for every abelian sheaf $G$ on $R$-Al $(m)$ we have

$$
\left(R^{n} \Sigma\right)(G)=R^{n}\left(\Sigma \circ j^{*}\right)\left(j_{*} G\right) .
$$

Proof. We know that $j_{*}$ is exact, and consequently $j^{*} M$ is injective whenever $M$ is an injective abelian sheaf on $R$-Al. We also know by (4.2) that if $G$ is flasque then $j_{*} G$ is also flasque. For such a flasque $G$, then, let

$$
0 \longrightarrow j_{*} G \longrightarrow M_{0} \longrightarrow M_{1} \longrightarrow \ldots
$$

be an injective resolution. Since $j_{*} G$ is flasque, the sequence is an exact sequence of functors [1, p. 39], and hence its restriction

$$
0 \longrightarrow G \longrightarrow j^{*} M_{0} \longrightarrow j^{*} M_{1} \longrightarrow \cdots
$$

stays exact and is an injective resolution of $G$. Applying $\Sigma$ to this and taking homology thus gives us $\left(R^{n} \Sigma\right)(G)$ as well as $R^{n}\left(\Sigma \circ j^{*}\right)\left(j_{*} G\right)$, so the two are equal for flasque $G$.

Take now any $G$ and resolve it by injectives, 


\section{$0 \longrightarrow G \longrightarrow I_{0} \longrightarrow I_{1} \longrightarrow \cdots$}

Each $I_{i}$ is flasque [1, p. 40], and hence by the previous step $R^{n}\left(\Sigma \circ j^{*}\right)\left(j_{*} I_{i}\right)$ equals $\left(R^{n} \Sigma\right)\left(I_{2}\right)$, which is zero. Therefore the exact sequence

$$
0 \longrightarrow j_{*} G \longrightarrow j_{*} I_{0} \longrightarrow j_{*} I_{1} \longrightarrow \cdots
$$

is a resolution by $\left(\Sigma \circ j^{*}\right)$-acyclics, and we can compute $R^{n}\left(\Sigma \circ j^{*}\right)\left(j_{*} G\right)$ by applying $\Sigma \circ j^{*}$ and taking homology. But since $j^{*} j_{*} I_{2} \cong I_{i}$, we get just $\left(R^{n} \Sigma\right)(G)$.

Thus the derived functors of $\Sigma \circ j^{*}$ applied to an $m$-based sheaf can be computed in the category of abelian sheaves on $R$-Al $(m)$. For example, let $F$ be a fixed abelian sheaf on $R$-Al $(m)$, and set $\Sigma(G)=\operatorname{Hom}(F, G)$. Then $\operatorname{Hom}\left(j_{*} F, L\right)=\operatorname{Hom}\left(F, j^{*} L\right)=\left(\Sigma \circ j^{*}\right)(L)$, so the theorem is applicable:

Corollary 7.2. Let $F$ and $G$ be abelian sheaves on $R$-Al $(m)$. Then $\operatorname{Ext}^{n}(F, G)=\operatorname{Ext}^{n}\left(j_{*} F, j_{*} G\right)$.

In this corollary we can in particular take $F$ to be the constant sheaf $Z$, which by (6.2) is $m$-based for any $m$. This gives us cohomology:

COROLlary 7.3. Let $G$ be an abelian sheaf on $R$-Al $(m)$. Then $H^{n}(G)=H^{n}\left(j_{*} G\right)$.

8. The functors $H^{n}\left(-, j_{*} G\right)$.

Theorem 8.1. Let $G$ be an abelian sheaf on $R$-Al $(m)$. Then the functors $A \mapsto H^{n}\left(A, j_{*} G\right)$ are m-based.

Proof. The groups $H^{n}(A, M)$ can be defined as the derived functors of $M \mapsto M(A)$, and they can be computed by flasque resolutions [1, p. 41]. As in (7.1), an injective resolution

$$
0 \longrightarrow G \longrightarrow I_{0} \longrightarrow I_{1} \longrightarrow \cdots
$$

yields a fiasque resolution

$$
0 \longrightarrow j_{*} G \longrightarrow j_{*} I_{0} \longrightarrow j_{*} I_{1} \cdots,
$$

and hence $H^{n}\left(A, j_{*} G\right)$ are the homology groups of the complex $j_{*} I_{i}(A)$. For $S$ in $R$-Al $(m)$ one can similarly compute the groups $H^{n}\left(S, j_{*} G\right)=H^{n}(S, G)$ as the homology groups of the complex $I_{2}(S)$. 
But each $j_{*} I_{i}(A)$ is the direct limit of $I_{i}(S)$ over all maps $S \rightarrow A$, giving us actually a direct limit of complexes. This limit, indexed by a filtered category, is exact; it therefore passes to homology, and $H^{n}\left(A, j_{*} G\right)$ is the direct limit of the $H^{n}\left(S, j_{*} G\right)$.

9. Ext functors and Ext sheaves. Let $L$ be an abelian sheaf on $R$-Al. If $A$ is any $R$-algebra, composing $L$ with the forgetful functor yields a sheaf $L_{A}$ on $A$-algebras, and it is then true [1, p. 45] that $H^{n}\left(L_{A}\right)$ is the same as $H^{n}(A, L)$. Comparing (7.2), (7.3), and (8.1), one might be tempted to conjecture that if $L$ and $M$ are $m$-based the functors $A \mapsto \operatorname{Ext}^{n}\left(L_{A}, M_{A}\right)$ should be $m$-based. But this is false even for $\mathrm{Ext}^{0}=\mathrm{Hom}$, as we now show.

ExAmPLe. Let $r$ be a cardinal. Let $G(B)=B$, and let $F(B)=$ $B^{(r)}$. Both $F$ and $G$ are countably based over the integers, $F$ being the direct sum of $r$ copies of $G$. Clearly $\operatorname{Hom}\left(F_{A}, G_{A}\right)=\operatorname{End}\left(G_{A}\right)^{r}$. But all units of $A$ give endomorphisms of $G_{A}$, and hence $A^{r}$ is contained in End $\left(G_{A}\right)^{r}$. Considering large $A$, we see that $A \mapsto$ Hom $\left(F_{A}, G_{A}\right)$ cannot be $m$-based for any $m$ less than $r$. Thus in general an increase in the cardinality is necessary.

Theorem 9.1. Let $F$ and $G$ be m-based abelian sheaves. Let $r$ be the cardinality of the disjoint union of all $F(S)$ for $S$ in $R$-Al $(m)$. Then the functors $A \mapsto \operatorname{Ext}^{n}\left(F_{A}, G_{A}\right)$ are all $r$-based.

Proof. We first introduce some special $m$-based sheaves and prove the result for them. Given a fixed $S$ in $R$-Al $(m)$, define a sheaf $\boldsymbol{Z}^{S}$ on $R$-Al by taking $\boldsymbol{Z}_{S}$ and extending by zero; explicitly, $\boldsymbol{Z}^{S}(B)$ is a direct sum of copies of $\boldsymbol{Z}(B)$, one copy for each map of $S$ to $B$. Clearly $\boldsymbol{Z}^{S}$ is an $m$-based sheaf, and $\operatorname{Hom}\left(\boldsymbol{Z}^{S}, L\right)=L(S)$ for any sheaf $L$. It is clear also that as sheaves on $A$-algebras we have $\left(\boldsymbol{Z}^{S}\right)_{A}=\boldsymbol{Z}^{A \otimes S}$, and hence Hom $\left(\left(\boldsymbol{Z}^{S}\right)_{A}, M\right)=M(A \otimes S)$ for every sheaf $M$ on $A$-Al. Therefore $\operatorname{Ext}^{n}\left(\left(Z^{S}\right)_{A}, M\right)=H^{n}(A \otimes S, M)$, and thus in particular $\operatorname{Ext}^{n}\left(\left(\boldsymbol{Z}^{S}\right)_{A}, G_{A}\right)=H^{n}(A \otimes S, G)$. But small algebras of the form $S^{\prime} \otimes S$ are cofinal among small algebras mapping to $A \otimes S$, and hence by (8.1) we know $H^{n}(A \otimes S, G)$ is the limit of $H^{n}\left(S^{\prime} \otimes S, G\right)$. In other words, the functors $A \mapsto \operatorname{Ext}^{n}\left(Z^{S}\right)_{A}, G_{A}$ ) are $m$-based.

Let $F$ now be an arbitrary $m$-based sheaf. For each $S$ in $R$-Al $(m)$ and each element of $F(S)$ there is a map $Z^{S} \rightarrow F$ sending 1 in $Z^{S}(S)$ to the prescribed element; let $L$ be the direct sum of all these $\boldsymbol{Z}^{s}$. Then $L$ is again an $m$-based sheaf. (In general we would have to sheafify, but since our coverings are finite the functor direct sum is a sheaf $[1$, p. 53].) The obvious map $L \rightarrow F$ is surjective at all $S$ in $R-\mathrm{Al}(m)$, and hence by $(2.1 \mathrm{f})$ is surjective 
everywhere. Let $K$ be its kernel, so that

$$
0 \longrightarrow K \longrightarrow L \longrightarrow F \longrightarrow 0
$$

is an exact sequence of $m$-based sheaves.

I claim that the cardinal $r$ which bounds the union of values of $F$ will do the same for $L$, and hence also for $K$. Indeed, we can identify $Z(B)$ with integer-valued functions on the finite sequences of orthogonal idempotents adding to 1 in $B$, and thus $\# Z(B) \leqq m$ for small $B$. Then $Z^{S}(B)$ is a sum indexed by maps $S \rightarrow B$, of which there are at most $2^{m}$, and $L(B)$ is a sum of $r$ such terms. By construction $r \geqq 2^{m}$, and hence $\# L(B) \leqq r$. There are only $2^{m}$ different $B$ in $R$-Al $(m)$, so $r$ also bounds the disjoint union.

Now for any $A$ in $R$-Al we have a long exact sequence

$$
\begin{aligned}
0 \longrightarrow \operatorname{Hom}\left(F_{A}, G_{A}\right) \longrightarrow \operatorname{Hom}\left(L_{A}, G_{A}\right) \longrightarrow \operatorname{Hom}\left(K_{A}, G_{A}\right) \\
\longrightarrow \operatorname{Ext}^{1}\left(F_{A}, G_{A}\right) \longrightarrow \operatorname{Ext}^{1}\left(L_{A}, G_{A}\right) \longrightarrow \operatorname{Ext}^{1}\left(K_{A}, G_{A}\right) \longrightarrow \cdots
\end{aligned}
$$

For each $T$ in $R-\mathrm{Al}(r)$ mapping to $A$ we get a similar exact sequence mapping to this one; since the limit over $T \rightarrow A$ is exact we get a limiting exact sequence mapping to this one. But $A \mapsto \operatorname{Ext}^{n}\left(L_{A}, G_{A}\right)$ is $r$-based, for it is a product of $r$ factors $\operatorname{Ext}^{n}\left(\left(\boldsymbol{Z}^{S}\right)_{A}, G_{A}\right)$ each of which is $m$-based. Thus we have isomorphism at all the middle terms, and hence an injection on the term $\operatorname{Hom}(F, G)$. But since the argument applies to $K$ with the same $r$, we have injection on the $\operatorname{term} \operatorname{Hom}(K, G)$, and hence bijection on $\operatorname{Hom}(F, G)$. Therefore there is similarly a bijection on $\operatorname{Hom}(K, G)$, and hence an injection on $\operatorname{Ext}^{1}(F, G)$, and so on.

Corollary 9.2. Let $\operatorname{Hom}(F, G)$ denote the sheaf $A \mapsto \operatorname{Hom}\left(F_{A}, G_{A}\right)$, and let $\operatorname{Ext}^{n}(F, G)$ be its derived sheaves. Then they are all $r$-based.

Proof. By [6, p. V-29] they are the sheafifications of the functors $A \mapsto \operatorname{Ext}^{n}\left(F_{A}, G_{A}\right)$.

For any basically bounded sheaves $F, G$ one can similarly show that $A \mapsto \operatorname{Morph}\left(F_{A}, G_{A}\right)$ is basically bounded.

\section{REFERENCES}

1. M. Artin, Grothendieck Topologies, Mimeographed notes, Harvard, 1962.

2. N. Bourbaki, Algèbre Commutative, Chapitres I et II. Hermann, Paris, 1961.

3. S. U. Chase and A. Rosenberg, Amitsur cohomology and the Brauer group, Mem. Amer. Math. Soc. No. 52 (1965), 34-79. 
4. M. Demazure, A. Grothendieck, et al., Schémas en Groupes (SGA 3), Inst. Hautes Études Sci., Paris, 1963/64.

5. P. Gabriel and F. Ulmer, Lokal präsentierbare Kategorien, Lecture Notes in Math. 221, Springer, Berlin, 1971.

6. J. Verdier, Topologies et faisceaux; in M. Artin et al., Cohomologie Étale des Schémas (SGA 4). Inst. Hautes Études Sci., Paris, 1963/64.

7. W. Waterhouse, Principal homogeneous spaces and group scheme extensions, Trans. Amer. Math. Soc., 153 (1971), 181-189.

Received April 24, 1973. Work on this paper was supported at various times by an NRC-ONR Research Associateship and by the contract NSF GP-25600.

CORNELL UNIVERSITY 


\section{PACIFIC JOURNAL OF MATHEMATICS}

\section{EDITORS}

RICHARD ARENS (Managing Editor)

University of California

Los Angeles, California 90024

\section{J. DugundJI}

Department of Mathematics University of Southern California Los Angeles, California 90007

D. Gilbarg and J. Milgram

Stanford University

Stanford, California 94305
University of Washington Seattle, Washington 98105

\section{ASSOCIATE EDITORS}
E. F. BECKENBACH
B. H. NeumanN
F. WolF
K. YoShIDA

\section{SUPPORTING INSTITUTIONS}

\author{
UNIVERSITY OF SOUTHERN CALIFORNIA \\ STANFORD UNIVERSITY \\ UNIVERSITY OF TOKYO \\ UNIVERSITY OF UTAH \\ WASHINGTON STATE UNIVERSITY \\ UNIVERSITY OF WASHINGTON \\ $\stackrel{*}{*} \stackrel{*}{*} \stackrel{*}{ }{ }^{*}$ AMERICAN MATHEMATICAL SOCIETY
}

The Supporting Institutions listed above contribute to the cost of publication of this Journal, but they are not owners or publishers and have no responsibility for its content or policies.

Mathematical papers intended for publication in the Pacific Journal of Mathematics should be in typed form or offset-reproduced, (not dittoed), double spaced with large margins. Underline Greek letters in red, German in green, and script in blue. The first paragraph or two must be capable of being used separately as a synopsis of the entire paper. Items of the bibliography should not be cited there unless absolutely necessary, in which case they must be identified by author and Journal, rather than by item number. Manuscripts, in triplicate, may be sent to any one of the editors. Please classify according to the scheme of Math. Reviews, Index to Vol. 39. All other communications should be addressed to the managing editor, or Elaine Barth, University of California, Los Angeles, California, 90024.

The Pacific Journal of Mathematics expects the author's institution to pay page charges, and reserves the right to delay publication for nonpayment of charges in case of financial emergency.

100 reprints are provided free for each article, only if page charges have been substantially paid. Additional copies may be obtained at cost in multiples of 50 .

The Pacific Journal of Mathematics is issued monthly as of January 1966. Regular subscription rate: $\$ 72.00$ a year (6 Vols., 12 issues). Special rate: $\$ 36.00$ a year to individual members of supporting institutions.

Subscriptions, orders for back numbers, and changes of address should be sent to Pacific Journal of Mathematics, 103 Highland Boulevard, Berkeley, California, 94708.

\section{PUBLISHED BY PACIFIC JOURNAL OF MATHEMATICS, A NON-PROFIT CORPORATION}

Printed at Kokusai Bunken Insatsusha (International Academic Printing Co., Ltd.), 270, 3-chome Totsuka-cho, Shinjuku-ku, Tokyo 160, Japan.

\section{Copyright (C) 1975 by Pacific Journal of Mathematics} Manufactured and first issued in Japan 


\section{Pacific Journal of Mathematics}

\section{Vol. 57, No. $2 \quad$ February, 1975}

Norman Larrabee Alling, On Cauchy's theorem for real algebraic curves with boundary .......

Daniel D. Anderson, A remark on the lattice of ideals of a Prüfer domain ..................

Dennis Neal Barr and Peter D. Miletta, A necessary and sufficient condition for uniqueness of

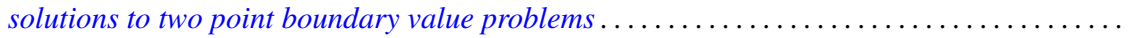

Ladislav Beran, On solvability of generalized orthomodular lattices . . . . . . . . . . ........

L. Carlitz, A three-term relation for some sums related to Dedekind sums . . . . . . . . . .....

Arthur Herbert Copeland, Jr. and Albert Oscar Shar, Images and pre-images of localization

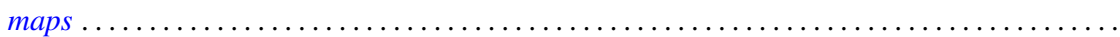

G. G. Dandapat, John L. Hunsucker and Carl Pomerance, Some new results on odd perfect

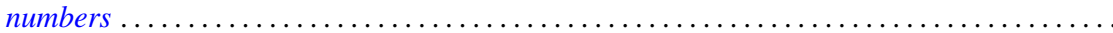

M. Edelstein and L. Keener, Characterizations of infinite-dimensional and nonreflexive

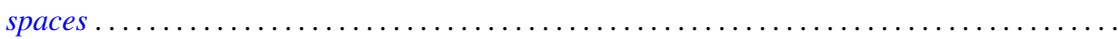

Francis James Flanigan, On Levi factors of derivation algebras and the radical embedding

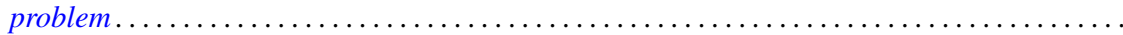

Harvey Friedman, Provable equality in primitive recursive arithmetic with and without

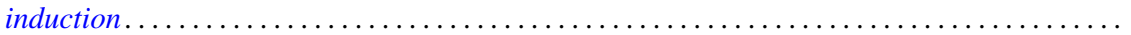

Joseph Braucher Fugate and Lee K. Mohler, The fixed point property for tree-like continua with

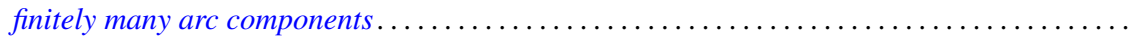

John Norman Ginsburg and Victor Harold Saks, Some applications of ultrafilters in

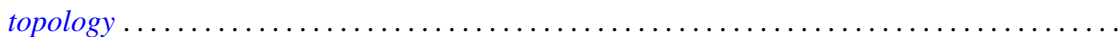

Arjun K. Gupta, Generalisation of a "square" functional equation .....................

Thomas Lee Hayden and Frank Jones Massey, Nonlinear holomorphic semigroups ..........

V. Kannan and Thekkedath Thrivikraman, Lattices of Hausdorff compactifications of a locally

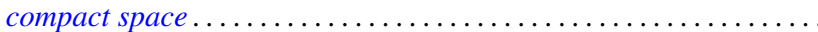

J. E. Kerlin and Wilfred Dennis Pepe, Norm decreasing homomorphisms between group

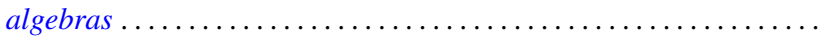

Young K. Kwon, Behavior of $\Phi$-bounded harmonic functions at the Wiener boundary ...

Richard Arthur Levaro, Projective quasi-coherent sheaves of modules .

Chung Lin, Rearranging Fourier transforms on groups...........................

David Lowell Lovelady, An asymptotic analysis of an odd order linear differential equation . . 4475

Jerry Malzan, On groups with a single involution .......................... 481

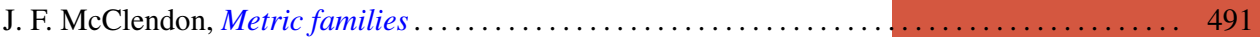

Carl Pomerance, On multiply perfect numbers with a special property .

Mohan S. Putcha and Adil Mohamed Yaqub, Polynomial constraints for finiteness of semisimple rings. .

Calvin R. Putnam, Hyponormal contractions and strong power convergence . . . . . . . . . 531

Douglas Conner Ravenel, Multiplicative operations in $\mathrm{BP} * \mathrm{BP} \ldots \ldots \ldots \ldots \ldots \ldots \ldots \ldots \ldots .539$

Judith Roitman, Attaining the spread at cardinals which are not strong limits . . . . . . . . . 545

Kazuyuki Saitô, Groups of *-automorphisms and invariant maps of von Neumann algebras . . . 553

Brian Kirkwood Schmidt, Homotopy invariance of contravariant functors acting on smooth

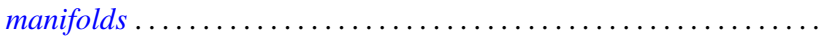

Kenneth Barry Stolarsky, The sum of the distances to $N$ points on a sphere.

Mark Lawrence Teply, Semiprime rings with the singular splitting property.

J. Pelham Thomas, Maximal connected Hausdorff spaces..............

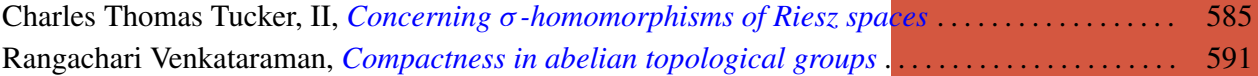

William Charles Waterhouse, Basically bounded functors and flat sheaves . . . . . . . . . . . 597

David Westreich, Bifurcation of operator equations with unbounded linearized part ......... 611

William Robin Zame, Extendibility, boundedness and sequential convergence in spaces of 